Two other resolutions concerned the international registration of trade marks. Another resolution provided that the validity of a 'patent of addition' should not necessarily be affected should the substantive patent be declared invalid or be revoked. In the light of the increase in modern scientific research, whether sponsored by Governments, by universities, or by firms, it is increasingly desirable that its practical or industrial applications should not only be carefully studied from a national aspect but also from an international aspect. The representatives at this Congress appreciated that if international trade is to be increased it is essential that harmony should be achieved between the laws of different countries relating to industrial property. The discussions at the Conference were sincere attempts to achieve this end, and the Conference assumed a further world-wide significance in view of the formation of the Economic Council of the United Nations Organisation.

\section{SIXTH INTERNATIONAL CONGRESS OF EXPERIMENTAL CYTOLOGY}

$\mathrm{T}$ HE Sixth International Congress of Experimental Cytology met in Stockholm during July 10-17; some four hundred members from twenty-three countries attended. It was of outstanding interest in several ways. But for the War, this Sixth Congress would have met in 1940 at Stockholm, so the international ties in this branch of science were here appropriately reformed. Secondly, the official scope of 'experimental cytology' has now been broadened. Under the late Prof. Rhoda Erdmann, research based on the tissue-eulture technique had taken a central position in pre-war meetings. Now, with the shift of the centre of gravity towards sub-microscopical biology, topies at this Congress ranged in ascending orders of magnitude from that of the large molecule to the whole organ.

To cover so wide a field, it was necessary in part to organise the Congress in separate sections, and here great tribute is due to the organising committee, and particularly to the general secretary, Dr. H. Hyden, for the admirable way in which the programme was arranged and carried out. The main sessions of the Congress generally took the form of symposia, at which recent work in each field was presented, mainly by the leading figures therein from New and old Worlds.

It was clear that there had disappeared one of the cleavages in pre-war biology, namely, that between the disciplines of biochemistry and microscopical investigation. The focal interest in the biological significance of the nucleic acids and the development of the technique of analysis by differential centrifugation have effectively contributed to bridge this gap.

The freedom with which different techniques are now being used in particular fields was well illustrated both by the papers on muscle proteins and in the microbiological sections. The results obtained by the different methods are by no means yet in complete agreement; but the interplay of the different points of view both in the papers and in discussion was of great interest.

The contribution of Swedish men of science to the success of the Congress was at least of equal importance to that of the membership. Laboratories in
Stockholm, Uppsala and elsewhere were freely open to visitors. Although Prof. Caspersson's new institute is not yet ready, there was a superb demonstration in the form of a series of panels which adequately expounded the work of his school. It was an experience deep with meaning to see the progress in science of a small country that has escaped the direct wastage of war.

The social side of the Congress was further memorable. Members were the guests of the city of Stockholm in the magnificent town hall on the shore of Lake Malar, and also, some days later, of the Biochemical Industrial Concern of Kärnbolaget.

The Congress concluded with a business session which refounded an International Society for Cell Biology, at which plans were announced for new international journals, of which one for cytological topics is to be edited from Sweden.

\section{ORGANISATION OF THE CAPILLARY CIRCULATION}

$\mathrm{W}$ HILE it is obvious that the capillary circulation is subject to local physiological control, there is much difference of opinion as to how this is achieved. In particular, it is still undecided whether or not the capillaries exhibit independent contractility and, if so, whether this is due to the presence of special perivascular contractile cells. Hitherto the capillary bed has been regarded as a network of capillaries of uniform structure fed by artierioles and drained by venules. Recent work, however, particularly by R. Chambers and B. W. Zwoifach, has shown that the capillary bed comprises several different types of vessels which can now be separately defined, and each of which plays a particular part in the total functional organisation of the capillary bed.

Chambers and $Z$ weifach have recently summarized their findings (Ann. New York Acad. Sci., 46, 683 ; 1946). Their observations were principally made on the rat's mesentery; but a similar pattern was found in subcutaneous tissue and skeletal muscle. They find that each arteriole is connected to a venule by one rather long straight vessel, the 'central channel' or 'arteriovenous channel' (not to be confused with arteriovenous anastomoses). The true capillaries arise as lateral offshoots from the proximal part of the central channel, form anastomosing networks and drain into the distal part of the central channel and also directly into venules. The proximal part of the central channel is called the 'metarteriole'; it is provided with muscle, and together with the arteriole is responsible for vasomotor control of the total blood flow through the capillary bed. The distal part of the central channel is non-muscular. Abrupt lateral branches from the metarteriole lead to the capillary networks. These lateral branches are muscular for a short distance. The muscular part is termed the 'precapillary' and acts as a sphincter controlling the admission of blood to the network of true capillaries beyond.

While the tissues are in a resting state there is relative ischæmia, the precapillary sphincters are closed and the blood flow is confined to the central channel. During tissue activity there is hyperæmia, the sphincters are open and blood flows through the whole capillary network. The true capillaries seem to exhibit no active vasomotor changes, their walls contain no adventitial contractile cells, neither do 
the endothelial cells themselves undergo active con. tractile changes in shape as has frequently been claimed.

This further analysis of the capillary bed, with its closer definition of the term 'capillary', may enable us to reconcile some of the conflicting views of pre. vious workers. For example, those who claim to have seen independent contraction of capillaries may, in fact, have been observing metarterioles or precapillaries. Chambers and Zweifach have established their findings in the case of the rat's mesentery, and they regard this pattern as the basic arrangement in all mammalian tissues, subject to local modifications in detail to suit the requirements of particular tissues. The frog's web and bat's wing, which have been so exhaustively studied by others, do not show the central channel type of circulation and are therefore regarded as atypical. It is still far from proved, however, that the pattern found in the rat's mesentery is typical of the mammalian capillary circulation in general. It is not unlikely that further patterns of capillary circulation remain to be discovered.

\section{ELECTRONIC EQUIPMENT AT THE ROYAL AIRCRAFT ESTABLISHMENT}

$\mathrm{V}$ ISITORS were recently invited by the Director of the Royal Aircraft Establishment to a demonstration of equipment developed by, or in conjunction with, the Establishment for the measurement of aircraft structural and power plant characteristics, both in the laboratory and in flight. Essentially, the equipment must convert the physical quantity being measured into a movement (unless the quantity itself involves a movement); convert the movement into the corresponding variation of an electrical parameter; and finally amplify and record the variation.

The pick-up gives either a change in electrical resistance, capacity, or inductance, or a small change of voltage generated either mechanically or piezoelectrically. The calibrating equipment displayed emphasized the importance of a full investigation of the factors controlling the dynamic performance of every type of pick-up, since there is an increasing demand for the accurate measurement of strains, pressures, and accelerations at high frequencies. Wire resistance strain gauges are now almost universally used for the measurement of a strain; condenser and piezo-electric pick-up units are preferred for the measurement of fluid pressure, especially at high frequency.

Resistance strain gauges are susceptible to spurious resistance changes, particularly important being those due to temperature and humidity changes such as occur in moving from ground-level to a high altitude. Using a temperature-compensating gauge in the circuit, temperature effects can be ignored, but the effect of a humidity change varies from gauge to gauge, so waterproofing of both the active and the compensating gauges is very important. A static strain of $10^{-6}$ can be measured using a standard type of gauge with a 'null' method and manual balancing of the bridge circuit, which takes about six seconds for each station. For more than 250 gauge positions, time is saved by automatic switching. High-speed rotary switching makes it possible to photograph in the laboratory up to 600 cathode ray tube records of steady signals in about five seconds, but further time is, of course, spent in preparing the results for examination. A pen-andink recording unit operated by the out-of-balance current takes ninety-six readings in about half a minute.

The resistance gauge has also been applied to determine acceleration, as the strain of a loaded thin-walled cylinder; pressure, as a strain due to the deformation of $a$ diaphragm or thin-walled cylinder; and force, as the strain of a short hollow cylinder in axial compression. The measuring element is usually of beryllium copper, which has a high proportional limit and is remarkably free from hysteresis. Future development includes the finding of more suitable gauge materials for high-temperature work.

Other types of gauge indicate the deflexion of one plate of an electrical condenser, or cause variation in the inductance and losses of two coils when a separating diaphragm, a common core, or a surround. ing copper ring is displaced. A piezo-electric pick-up for the measurement of blast pressure consists of two pairs of crystals and two pistons to which the pressure is applied. Generator type vibration pick-ups were exhibited, measuring the movement of a vibrating body with respect to a stationary magnet; but as yet it has been found difficult to design a small, robust vibration pick-up unit with an undamped natural frequency less than 5 cycles per second, so that, below about 20 c.p.s., frequency and phase errors cannot be neglected.

To change alternating variations in resistance, capacitance or inductance into corresponding voltage changes before amplification and recording, the pick-up usually forms one or more arms of an A.c. bridge, the impedance change modulating the carrier-voltage. The output may be amplified by a stable A.C. coupled amplifier which has only to deal with the frequency range determined by the modulation sidebands. The modulated signal is demodulated immediately before recording, preferably in a phasesensitive demodulator which gives an output the sign or phase of which is directly related to the phase of the carrier voltage and so differentiates between increase and decrease of pick-up impedance.

Freedom from instability, distortion, and microphony, and adequate response to low-frequency signals are desirable characteristics of the amplifier. Fairly simple direct-coupled amplifiers of adequate stability and gain are in use on ground equipment. Six-channel units photographing $3 \frac{1}{2}$-in. cathode ray tubes are available with amplifiers of voltage gain 20,000 and frequency response zero to 10,000 cycles per second. An alternative to the bridge circuit for capacitance or inductance units is the frequency- or amplitude-modulation resonant circuit, using a carrier frequency of one megacycle per second or higher. Although more complicated, this type of circuit has the advantage that, since attenuation and phase distortion introduced in passing between the oscillator and discriminator are unimportant, much of the equipment can be placed to avoid microphony.

It is essential for rapid recording from a large number of stations and for determining phase relations to use multi-channel equipment for flight work. The restrictions imposed by flight conditions have caused much attention to be given to the simple and compact bifilar or coil type electromagnetic vibration galvanometers, although the galvanometer 